\title{
Efectos del pastoreo sobre el banco de semillas germinable y la vegetación establecida en pastizales de montaña del centro de Argentina
}

\author{
Grazing effects on the germinable seed bank and standing vegetation in mountain \\ grasslands from central Argentina
}

SEBASTIÁN MÁRQUEZ', GUILLERMO FUNES ${ }^{1 *}$, MARCELO CABIDO ${ }^{1} \&$ EDUARDO PUCHETA $^{1,2}$

${ }^{1}$ Instituto Multidisciplinario de Biología Vegetal (IMBIV-CONICET) y Cátedra de Biogeografía, Facultad de Ciencias Exactas, Físicas y Naturales, Universidad Nacional de Córdoba, CC 495, 5000 Córdoba, Argentina

${ }^{2}$ Departamento de Geofísica, Astronomía y Biología, Facultad de Ciencias Exactas, Físicas y Naturales,
Universidad Nacional de San Juan, San Juan, Argentina
*Correspondencia a: e-mail: gfunes@imbiv.unc.edu.ar

RESUMEN

Se estudió el efecto del pastoreo sobre el banco de semillas y la vegetación establecida en pastizales naturales de montaña, en Pampa de Achala, Córdoba, Argentina. Se analizó el banco de semillas germinable a dos profundidades (0-5 y 5-10 cm), en cinco réplicas de sitios pastoreados a carga moderada-intensa y otros cinco de sitios excluidos al pastoreo por 10 años. Se registró la frecuencia de las especies presentes en la vegetación establecida y la abundancia de las especies en el banco de semillas. El pastoreo no produjo cambios significativos en el número de especies de la comunidad establecida, pero sí produjo un incremento en la diversidad de especies debido a una disminución de la frecuencia de algunos pastos perennes como Deyeuxia hieronymi y Festuca tucumanica. Se registraron sólo tres especies exóticas en los pastizales estudiados. El pastoreo no produjo cambios significativos en la riqueza ni en la diversidad de especies del banco de semillas. La densidad total de semillas en el banco tampoco cambió significativamente por efecto del pastoreo. Sin embargo, la densidad de semillas de una especie de gramínea anual, Muhlenbergia peruviana, aumentó, mientras que la densidad de semillas de una especie de pasto perenne de gran tamaño, Deyeuxia hieronymi, disminuyó significativamente con el pastoreo. Las semillas contenidas en la hojarasca de los sitios excluidos al pastoreo comprendió el $20 \%$ del total de especies y el $43 \%$ de la abundancia total de semillas del banco. El análisis de ordenamiento de las parcelas en función de la composición florística de la vegetación y el banco de semillas germinable, evidenció una mayor segregación de las parcelas en función del pastoreo y, en menor medida, debido a diferencias entre vegetación y banco de semillas. Tanto en los sitios pastoreados como en los sitios excluidos al ganado predominaron las especies con banco de semillas transitorio, seguidas de especies con banco de semillas persistente a corto y a largo plazo. El pastoreo, o su ausencia, no modifica la abundancia en el banco de semillas de la mayoría de las especies, sin embargo, las dos especies que sí cambian de manera importante su presencia en el banco, responden a dos estrategias opuestas, según lo observado por otros autores, una domina la productividad primaria del ecosistema en ausencia del pastoreo, la otra, ocuparía un papel importante en la cicatrización de huecos a la escala de microparche en sitios muy perturbados.

Palabras clave: pastoreo, exclusión, vegetación establecida, diversidad, banco de semillas transitorio, banco de semillas persistente.

\begin{abstract}
We studied the effect of grazing on the standing vegetation and the germinable soil seed bank in natural mountain grasslands at Pampa de Achala, Córdoba Province, Argentine. We analyzed the germinable seed bank at two depths (05 and $5-10 \mathrm{~cm}$ ) in five replicates from sites grazed at a moderate to intense grazing intensity, and in five replicates from sites excluded from herbivores during the last 10 years. We recorded species frequency on the standing vegetation and species abundance in the germinable seed bank. Grazing did not produce significant changes in the number of species of the standing vegetation, but produced an increase in species diversity due to a decrease in the dominance of perennial grasses such as Deyeuxia hieronymi and Festuca tucumanica. We registered only three alien species in the grasslands under study. Grazing did not produce significant changes in the seed bank richness and diversity. Average seed bank density significantly did not change after grazing, however, the density of the annual grass Muhlenbergia peruviana increased, while that of the tussock grass Deyeuxia hieronymi decreased in grazed plots. Seeds present in litter at the excluded sites comprised $20 \%$ of the total number of species and $43 \%$ of the total seed density. Ordination analysis, taking into account floristic composition of standing vegetation and the germinable seed bank, showed higher segregation between grazed-ungrazed samples than samples from vegetation and from the seed bank. At both grazed and protected sites, there were dominance of species with transient seed banks, followed by species with short and long persistent seed banks, respectively. Grazing, or its absence, did not modify plant species abundance of most species at
\end{abstract}


the germinable seed bank, moreover, the two species that changed their seed density showed two opposite plant strategies, as observed previously: one dominates primary productivity in absence of grazing, the another could play an important role in gap restoration at a micro patch scale in heavy grazed sites.

Key words: grazing, exclusion, standing vegetation, diversity, transient seed bank, persistent seed bank.

\section{INTRODUCCIÓN}

El pastoreo representa un factor clave en relación a la estructura y al funcionamiento de las comunidades de pastizal, modificando su composición florística y diversidad (Belsky 1992, Milchunas \& Lauenroth 1993, Leps et al. 1995), su productividad primaria neta aérea (McNaughton 1979, Sala et al. 1981, Pandey \& Singh 1992, Redmann 1992), y sus características potenciales de regeneración, tanto vegetativa como a partir del banco de semillas (O'Connor 1991, Bertiller 1992, Kinukan \& Smeins 1992, Milberg 1995, Bertiller 1996, Ungar \& Woodell 1996, Bertiller \& Aloia 1997).

Diferentes aspectos del banco de semillas como su densidad y composición (Peco et al. 1998), su persistencia en el suelo (Bakker et al. 1996, Mc Donald et al. 1996), y su similitud florística con la vegetación establecida (Ortega et al. 1997, Peco et al. 1998), permiten comprender mejor la dinámica de la comunidad de plantas y su respuesta frente a las perturbaciones (Van Der Valk $\&$ Pederson 1989, Bakker et al. 1996). Por otra parte el pastoreo, a través de su influencia sobre la supervivencia de las plantas y sobre la producción de semillas (Grime 1979, Kinucan \& Smeins 1992), puede afectar la densidad y composición de los bancos de semillas (Watt \& Gibson 1988, Oesterheld \& Sala 1990), modificar la persistencia de las semillas en el banco (Bakker et al. 1996), y producir cambios relativos entre la abundancia de las especies de la vegetación establecida y del banco de semillas (O'Connor 1991, Bertiller 1992, Milberg 1995). Los resultados documentados hasta el momento en relación al efecto del pastoreo sobre la formación de bancos de semillas en diferentes pastizales del mundo son contradictorios, por lo que no se puede establecer aún un único patrón. Por ejemplo, tanto Milberg (1995), en pastizales templado-fríos de Europa, como Ortega et al. (1997), en pastizales mediterráneos de montaña, encontraron que el pastoreo no produce cambios en la riqueza de especies en el banco. Sin embargo, Donelan \& Thompson (1980), Gibson \& Brown (1991) y McDonald et al. (1996) en pastizales similares a los mencionados más arriba, sugieren que el pastoreo produce un enriquecimiento de especies en el banco de semillas. Por otro lado, se ha sugerido que el pastoreo disminuye la densidad de semillas en el suelo (Bertiller 1992, O’Connor \& Pickett
1992, Ortega et al. 1997). Aunque Milberg \& Hansson (1993) y Milberg (1995) no observaron efectos del pastoreo sobre la densidad de semillas en el banco.

Los pastizales de altura de las Sierras de Córdoba han estado sujetos al pastoreo por animales domésticos desde la ocupación española hace más de 300 años (Díaz et al. 1994a), y soportan actualmente un régimen de ganadería extensiva, con cargas moderadas a altas de ganado vacuno y equino. Algunas respuestas de la comunidad vegetal frente a la acción de los herbívoros domésticos han sido estudiadas previamente, como cambios en su fenología (Díaz et al. 1994b), en su composición florística y diversidad (Pucheta \& Cabido 1992, Pucheta et al. 1992, 1997, 1998a, 1998 b), y en su biomasa y productividad primaria neta aérea (Sipowicz et al. 1978, Pucheta et al. 1998a, 1998b). En estos estudios previos se sugiere que la herbivoría es un factor incorporado al sistema, sin embargo, hasta el momento no se conoce cuál es el efecto del pastoreo sobre el potencial de regeneración de estas comunidades de pastizales naturales a partir del banco de semillas.

El objetivo del presente trabajo fue evaluar el efecto del pastoreo sobre: (i) la densidad total del banco de semillas, (ii) la similitud entre la composición florística de la vegetación y del banco de semillas y (iii) los tipos de banco de semillas según su persistencia en el suelo.

\section{MATERIALES Y MÉTODOS}

\section{Descripción del área}

El estudio se realizó sobre pastizales naturales en la altiplanicie granítica de Pampa de Achala ( $31^{\circ}$ $24^{\prime}-31^{\circ} 50^{\prime} \mathrm{S}, 64^{\circ} 45^{\prime}-64^{\circ} 52^{\prime}$ O O), en las Sierras Grandes, provincia de Córdoba, Argentina, una altitud media superior a los $2.000 \mathrm{~m}$. El clima en el área es templado frío con veranos cortos y frescos, e inviernos fríos. La temperatura media de invierno es de $5^{\circ} \mathrm{C}$, mientras que la de verano es de $11,4^{\circ} \mathrm{C}$. Las precipitaciones están concentradas entre noviembre y marzo, alcanzando un valor promedio anual de $850 \mathrm{~mm}$ para 12 años de registros. No hay ningún mes totalmente libre de heladas y suelen ocurrir algunas nevadas (Cabido et al. 1987).

Desde el punto de vista fitogeográfico, el área de estudio se considera comprendida en el subpiso 
superior de pastizales de altura de las Sierras de Córdoba, perteneciente al Chaco Serrano (sensu Luti et al. 1979), aunque actualmente se considera que la flora del área tiene afinidades con la flora andina y de otros sectores montañosos de la Argentina (Funes \& Cabido 1995, Cabido et al. 1998).

El presente estudio se concentró en dos de los diversos estados sucesionales que pueden presentar los pastizales naturales de las Sierras Grandes (Pucheta et al. 1997), representados por los pajonales de Deyeuxia hieronymi y Festuca tucumanica, y por los céspedes de pastoreo de Alchemilla pinnata, Carex fuscula y Relbunium richardianum. Los pajonales y los céspedes de pastoreo constituyen las comunidades más extendidas en la región y se componen de especies perennes nativas con escasos taxa exóticos (Cabido et al. 1987, Pucheta \& Cabido 1992, Pucheta et al. 1992, 1998a, 1998b).

\section{Diseño del muestreo}

Se consideraron dos tratamientos: uno pastoreado a una carga ganadera moderada a intensa (1 vaca 3-4 ha-1), y uno excluido al pastoreo por 10 años, donde el ganado fue retirado luego del último trazado de la Ruta Nacional 20, situación similar a la utilizada por Noy-Meir et al. (1989). Para cada tratamiento se tuvieron en cuenta cinco réplicas de $20 \times 20 \mathrm{~m}$.

\section{Vegetación establecida}

Para determinar el efecto del pastoreo sobre la composición florística de la vegetación establecida se utilizó un método de muestreo de puntos al azar (Krebs 1985). Para esto, en cada una de las 5 réplicas de los tratamientos se ubicaron aleatoriamente 50 agujas donde se registró la presencia de todas las especies de plantas vasculares.

\section{Banco de semillas}

Para determinar el efecto del pastoreo sobre la composición y abundancia del banco de semillas, en cada una de las réplicas se tomó una muestra de suelo compuesta de 10 submuestras con un sacabocados de $35 \mathrm{~mm}$ de sección (Funes et al. 1999). Las submuestras se tuvieron en cuenta para minimizar los errores relacionados con la gran heterogeneidad espacial que presentan los bancos de semillas (Thompson 1986). Se tuvieron en cuenta los primeros $10 \mathrm{~cm}$ de profundidad del suelo. La columna de suelo obtenida de las submuestras se dividió en dos profundidades $(0-5$ y $5-10 \mathrm{~cm})$ con el objeto de determinar la distribución vertical de las semillas en el suelo y asignar las especies presentes a un tipo de estrategia de banco de semillas, sensu Thompson et al. (1997). El muestreo se realizó en el mes de mayo de 1997 luego de la lluvia de semillas, a los efectos de captar la mayoría de las especies presentes en el banco y aquéllas cuyas semillas tienen una corta persistencia en el suelo. Este modelo de clasificación de los bancos de semillas (transitorio, persistente a corto o a largo plazo) está basado en la distribución vertical de las semillas en el suelo y en la presencia de la especie en la comunidad establecida (Thompson et al. 1997).

Las especies con banco de semillas transitorios son aquéllas que están sólo presentes en la vegetación establecida o en la capa superficial del suelo. Especies con banco de semillas persistentes por corto tiempo son aquéllas cuyas semillas se presentan en mayor abundancia en la capa superficial del suelo y en menor medida a mayor profundidad. Estas semillas persisten en el suelo hasta 5 años y son importantes en el mantenimiento de las poblaciones. Las especies con banco de semillas persistentes por largo tiempo poseen mayor o igual densidad de semillas en la capa inferior del suelo en relación a la capa superior. Éstas, persisten en el suelo por más de 5 años y son importantes en la regeneración de comunidades de plantas luego de un disturbio intenso, por lo que son muy consideradas en la ecología de la restauración (Bakker et al. 1996, Thompson et al. 1997).

Para determinar si la hojarasca acumulada sobre el suelo retiene semillas germinables, en cada réplica del tratamiento excluido al ganado (en el tratamiento pastoreado no se acumula hojarasca) se colectó una muestra compuesta por 10 submuestras de $10 \times 10 \mathrm{~cm}$ de toda la hojarasca depositada sobre el suelo.

Las muestras se pasaron a través de un tamiz de 2,5 mm de malla con el fin de eliminar fragmentos de plantas y pedregullos (Ter Heerdt et al. 1996, Funes et al. 1999), luego se expusieron a baja temperatura $\left(5^{\circ} \mathrm{C}\right)$ por un período de 35 días con la finalidad de romper la latencia de la mayor cantidad de semillas posible (Ter Heerdt et al. 1996). El material resultante se colocó en bandejas plásticas de $15 \times 15 \mathrm{~cm}$ sobre un soporte de 2 $\mathrm{cm}$ de arena esterilizada en autoclave a $120{ }^{\circ} \mathrm{C}$, para evitar la germinación de semillas indeseables o la proliferación de hongos. Las bandejas se mantuvieron en invernadero bajo condiciones controladas, a una temperatura media diaria de 25 
${ }^{\circ} \mathrm{C}$, e hidratadas diariamente a capacidad de campo. Se utilizaron cinco bandejas como controles, conteniendo sólo arena estéril, con el propósito de detectar la posible invasión de semillas exógenas. Las bandejas se distribuyeron sobre mesas siguiendo un diseño completamente aleatorizado.

El tamaño y composición del banco de semillas se evaluó mediante la identificación y conteo de las plántulas que emergieron de las muestras de suelo y hojarasca. Dichas plántulas se mantuvieron en el invernáculo hasta su correcta identificación taxonómica.

\section{Análisis de los datos}

Para determinar las principales tendencias de variación en la composición florística y abundancia de las especies en el banco de semillas y en la vegetación establecida, en relación con el pastoreo, se realizó un análisis de ordenamiento de las réplicas de ambos tratamientos (DCA, Hill \& Gauch 1980). Los valores de frecuencia de las especies en la vegetación establecida y de abundancia de las presentes en el banco de semillas en la matriz original, se transformaron en valores de abundancia relativa para evitar distorsiones. Se comparó, además, la composición de la vegetación y el banco de semillas de cada tratamiento por medio de coeficientes de similitud de Sørensen y se estimaron índices de diversidad de Shannon (Krebs 1985).

Las posibles diferencias debidas al efecto del pastoreo sobre el tamaño del banco de semillas total y de cada especie, se evaluaron mediante pruebas no paramétricas de Kolmogorov-Smirnov (Sokal \& Rohlf 1995) con la ayuda del programa SPSS (Norusis 1992).
Nomenclatura de las especies según Zuloaga \& Morrone (1996, 1999).

\section{RESULTADOS}

\section{Vegetación establecida}

Se registraron 97 especies de plantas vasculares en la comunidad (vegetación establecida + banco de semillas). Las especies más frecuentes en los sitios pastoreados fueron Alchemilla pinnata, Muhlenbergia peruviana, Carex fuscula y Agrostis montevidense, mientras que las más frecuentes en los sitios excluidos al ganado fueron Deyeuxia hieronymi, Poa stuckertii, y Alchemilla pinnata. Algunas especies como Muhlenbergia peruviana o Stipa nidulans son exclusivas de los sitios pastoreados, otras, como Poa stuckertii o Festuca tucumanica lo son en los sitios excluidos al pastoreo. En la vegetación establecida se registraron tres especies exóticas (Poa annua, Taraxacum officinale y Rumex acetosella), todas de amplia distribución (Anexo 1).

No se observaron diferencias significativas en la riqueza de especies entre los tratamientos, pero la diversidad de especies fue significativamente mayor en el tratamiento pastoreado (Tabla 1). Se observó una similitud (Sørensen) de especies entre tratamientos del $37 \%$.

Riqueza y densidad del banco de semillas germinable

Se identificaron 58 especies en el banco. Muhlenbergia peruviana, Pycreus rivularis, Juncus dichotomus y Alchemilla pinnata, fueron las especies más abundantes en los bancos de

TABLA 1

Riqueza (S) y diversidad (H’) de especies ( \pm error estándar, $\mathrm{n}=5$ ) de la vegetación establecida y el banco de semillas en los sitios excluido y pastoreado; $\mathrm{P}_{\mathrm{S}}$ y $\mathrm{P}_{\mathrm{H}}$ : valores de probabilidad asociados a la prueba de Kolmogorov-Smirnov para la riqueza y diversidad de especies entre tratamientos

Species richness $(\mathrm{S})$ and diversity $\left(\mathrm{H}^{\prime}\right)( \pm$ standard error, $\mathrm{n}=5$ ) both in the standing vegetation and in the seed bank in excluded and grazed sites; $\mathrm{P}_{\mathrm{S}}$ y $\mathrm{P}_{\mathrm{H}}$ : values of probability associated with the Kolmogorov-Smirnov test for species richness and diversity between treatments

\begin{tabular}{lcccccc}
\hline & \multicolumn{2}{c}{ Excluido } & \multicolumn{2}{c}{ Pastoreado } & \multicolumn{2}{c}{$\mathrm{K}-\mathrm{S}$} \\
& $\mathrm{S}$ & $\mathrm{H}^{\prime}$ & $\mathrm{S}$ & $\mathrm{H}$ & $\mathrm{P}_{\mathrm{S}}$ & $\mathrm{P}_{\mathrm{H}^{\prime}}$ \\
\hline Vegetación establecida & $18 \pm 1,64$ & $0,89 \pm 0,07$ & $28,2 \pm 1,74$ & $2,46 \pm 0,13$ & 0,082 & 0,013 \\
Banco de semillas & $14,2 \pm 2,24$ & $1,97 \pm 0,12$ & $18,8 \pm 2,42$ & $1,60 \pm 0,24$ & 0,329 & 0,819 \\
\hline
\end{tabular}




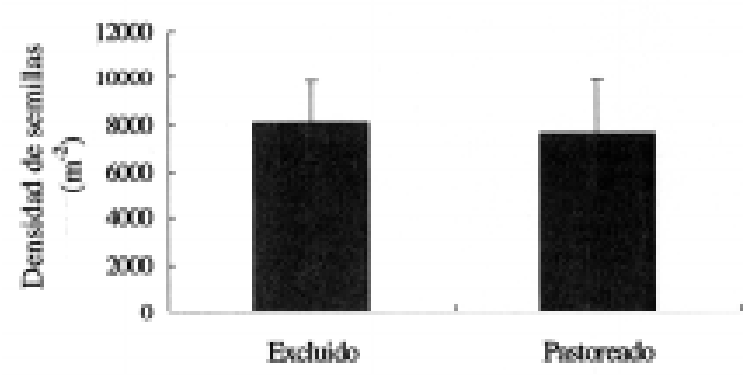

Fig. 1: Densidad total del banco de semillas ( semillas $\left.\mathrm{m}^{-2}, 0-10 \mathrm{~cm}\right)$ y error estándar $(\mathrm{n}=5)$ en los sitios excluido y pastoreado.

Seed bank density (seeds $\mathrm{m}^{-2}, 0-10 \mathrm{~cm}$ ) and standard error $(\mathrm{n}=5)$ in excluded and grazed sites.

semillas de los sitios pastoreados, mientras que en los sitios excluidos al pastoreo, las más abundantes fueron Deyeuxia hieronymi, Carex sp., Gamochaeta coarctata, Juncus dichotomus, Pycreus rivularis y Alchemilla pinnata.

No se observaron diferencias significativas en la riqueza ni en la diversidad de especies entre ambos tratamientos (Tabla 1). La presencia de especies exóticas en el banco fue baja, encontrándose sólo cinco especies (Linaria texana, Rumex acetosella, Silene antirrhina, Taraxacum officinale y Triodanis perfoliata v. Biflora) (Anexo 1).
No se observaron diferencias significativas en la densidad total del banco de semillas entre tratamientos ( $\mathrm{P}=0,819$, Fig. 1). La hojarasca retuvo el $43 \%$ (3.484 \pm 917 semillas $\mathrm{m}^{-2}$ ) de las semillas encontradas en los sitios excluidos al pastoreo. A pesar de la similitud en la densidad de semillas entre tratamientos, el pastoreo modificó la abundancia de dos especies: Muhlenbergia peruviana aumentó significativamente bajo pastoreo $(\mathrm{P}=$ 0,003), mientras que lo contrario ocurrió con Deyeuxia hieronymi $(\mathrm{P}=0,042)$. Estas especies mostraron el mismo comportamiento en la comunidad establecida $(\mathrm{P}=0,013$ y $\mathrm{P}=0,011$, respectivamente).

\section{Banco de semillas y vegetación establecida}

Del total de especies registradas (97, Anexo 1) en los pastizales de Pampa de Achala, sólo 26 son comunes a la vegetación y al banco de semillas. El análisis de ordenamiento de los sitios pastoreados y excluidos al pastoreo en función de la composición florística de la vegetación y del banco de semillas, evidenció mayores diferencias florísticas entre las parcelas bajo los efectos del pastoreo (eje 1, Fig. 2), y en menor medida reflejó diferencias entre la vegetación establecida y el banco de semillas (eje 2, Fig. 2). Además, dentro

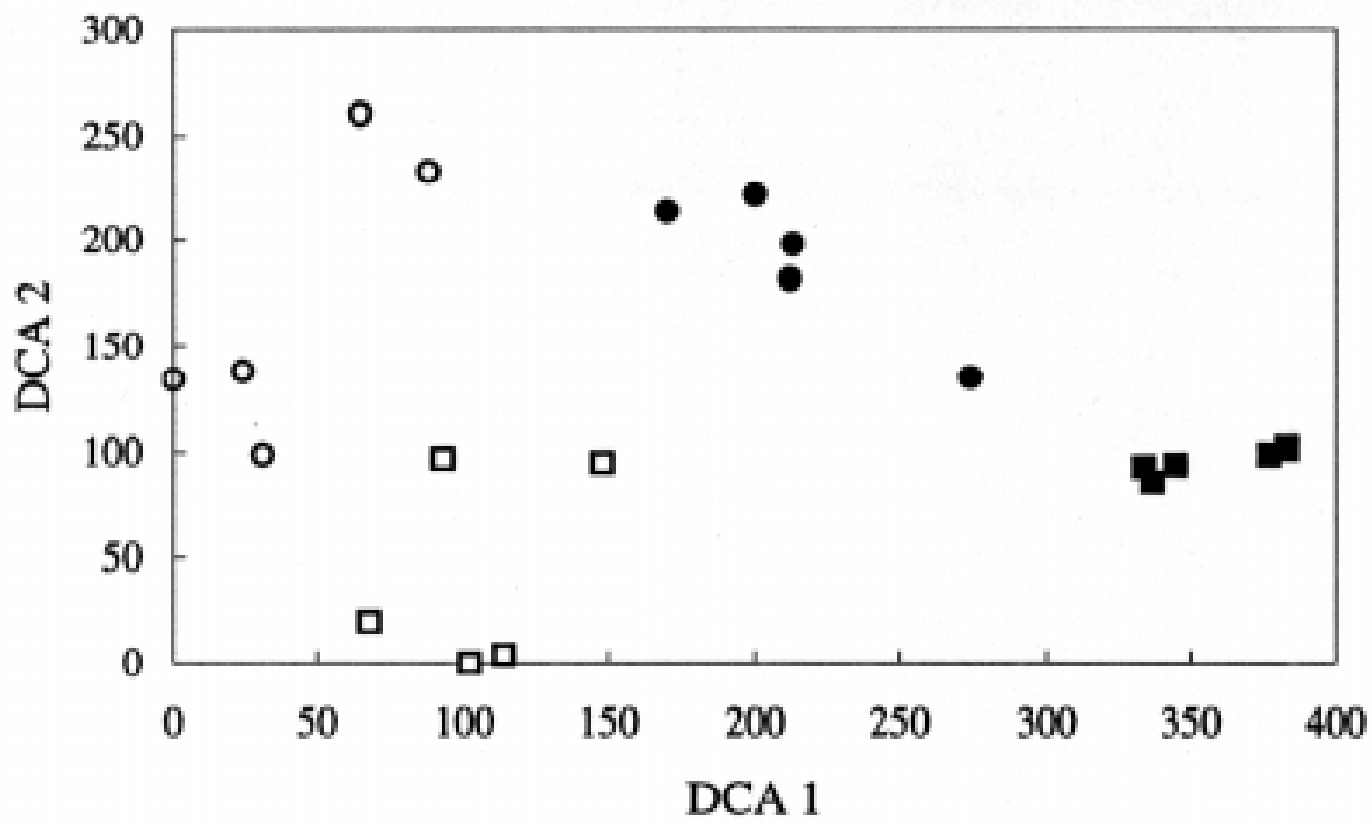

Fig. 2: Ordenamiento de las réplicas de cada tratamientos considerando la vegetación establecida y el banco de semillas, según eje 1 y eje 2 de DCA. $\mathbf{m}$ : vegetación establecida excluido; $\square$ : vegetación establecida pastoreado; $\bullet$ : banco de semillas excluido y $\bigcirc$ : banco de semillas pastoreado.

Ordination of replicates for each treatment considering the standing vegetation and seed bank, according axis 1 and axis 2 of DCA. $\square$ : non-grazed standing vegetation; $\square$ : grazed standing vegetation; $\bigcirc$ : non-grazed seed bank and $\bigcirc$ : grazed seed bank. 
TABLA 2

Porcentaje de especies que adoptan diferente estrategia de banco de semillas en los sitios excluido y pastoreado

Percentages of species showing different seed bank strategies in excluded and grazed sites

\begin{tabular}{lccc}
\hline & Transitorio & Persistente a corto plazo & Persistente a largo plazo \\
\hline Excluido $(\mathrm{n}=74)$ & 75 & 18 & 7 \\
Pastoreado $(\mathrm{n}=77)$ & 69 & 17 & 14 \\
\hline
\end{tabular}

de cada tratamiento los valores de similitud encontrados entre vegetación y banco de semillas fueron similares, $37 \%$ para sitios excluidos y 36 $\%$ para sitios pastoreados.

\section{Clasificación de los bancos de semillas}

En ambos tratamientos predominaron las especies con banco de semillas transitorio, seguidas por especies con banco de semillas persistente a corto y a largo plazo (Tabla 2). El pastoreo produjo un incremento de especies con banco de semillas persistente a largo plazo (Tabla 2). Algunas especies adoptaron diferente estrategia de banco de semillas frente al pastoreo o su exclusión (Anexo 1).

\section{DISCUSIÓN}

\section{Vegetación establecida}

En el pastizal natural estudiado el pastoreo no produjo cambios florísticos importantes, aunque sí un incremento en la diversidad de especies debido a una disminución de la dominancia de algunas de ellas. Resultados similares frente al pastoreo moderado a intenso por ganado doméstico se han observado en áreas próximas a la de estudio (Pucheta \& Cabido 1992, Pucheta et al. 1992, 1998a, 1998b). Por otra parte, en pastizales de la Pampa argentina (Sala et al. 1986, Chaneton \& Facelli 1991) y de otros lugares del mundo (Milchunas et al. 1988, Wilson 1990), se ha observado que el pastoreo produce importantes cambios florísticos a través de un enriquecimiento de especies exóticas. En los pastizales estudiados, sólo se han observado algunas especies exóticas cosmopolitas a intensidades moderadas a altas de pastoreo (Pucheta \& Cabido 1992, Pucheta et al. 1992, 1998a, 1998b), aunque en pastizales similares y próximos al área de estudio, Díaz et al. (1994a) registraron un gran número de especies exóticas luego de una perturbación mucho más severa que el pastoreo, como lo es el arado.

\section{Riqueza y densidad del banco de semillas}

En los pastizales estudiados, el pastoreo por ganado doméstico no produjo cambios significativos en la riqueza ni en la diversidad de especies del banco de semillas, en este sentido, los resultados encontrados en diferentes ecosistemas de pastizal no siguen un patrón único. Tanto Milberg (1995), en pastizales templado-fríos de Europa, como Ortega et al. (1997), en pastizales mediterráneos de montaña, encontraron resultados similares a los documentados en el presente estudio. Sin embargo, Donelan \& Thompson (1980), Gibson \& Brown (1991) y McDonald et al. (1996) en otros pastizales templados sugieren que el pastoreo produce un enriquecimiento de especies en el banco de semillas. La aparición o el enriquecimiento de especies exóticas en los bancos de semillas ha sido explicado por niveles altos de perturbación (Graham \& Hutchings 1988, Gilfedder \& Kirkpatric 1993). La escasa ocurrencia de especies exóticas en el banco de semillas estudiado, sumado al reducido efecto del pastoreo sobre la composición florística de la vegetación establecida, sugieren que el pastoreo no estaría actuando como una perturbación severa, conclusiones similares a las alcanzadas por Díaz et al. (1994a) y Pucheta et al. (1998a, 1998b) cuando analizan las respuestas de la vegetación frente al pastoreo por ganado doméstico en la misma área de estudio.

El pastoreo tampoco produjo cambios significativos en la densidad del banco de semillas. Este hecho ha sido observado en situaciones similares por Milberg \& Hansson (1993) y por Milberg (1995). Por el contrario, otros autores (Bertiller 1992, O'Connor \& Pickett 1992, Ortega et al. 1997), hallaron un menor número de semillas en los bancos de sitios pastoreados, coincidente probablemente con una disminución de la cobertura 
de plantas y un incremento en la predación de semillas por el ganado.

En los pastizales naturales de Pampa de Achala, Pucheta et al. (1998a) observaron que la exclusión prolongada del pastoreo promueve la acumulación de una cantidad importante de hojarasca sobre el suelo $\left(882,4 \pm 85,5 \mathrm{~g} \mathrm{~m}^{-2}\right)$, en contraste con lo registrado en áreas pastoreadas $(15,5 \pm 2 \mathrm{~g}$ $\mathrm{m}^{-2}$ ). En el presente estudio se observó que la hojarasca, presente sólo en los sitios excluidos al pastoreo, retuvo el $43 \%$ de las semillas totales del banco, poniendo de manifiesto su papel en la captación de semillas, como lo sugiriera Jutila (1998). La acumulación de hojarasca en los sitios excluidos al ganado permitió un incremento significativo en el número de semillas de la especie dominante, Deyeuxia hieronymi, hecho que hace presumir que esta especie se estaría regenerando a partir del banco de semillas sólo en algunos parches. En sitios excluidos al ganado, similares a los del presente estudio, Pucheta et al. (1998a) observaron que D. hieronymi, junto a Festuca tucumanica, representan casi la totalidad de la productividad primaria neta aérea, lo que sugiere que estas especies controlarían los procesos ecosistémicos en ausencia prolongada de los herbívoros.

En sitios pastoreados, donde el suelo carece de una cubierta de hojarasca, se observó un incremento significativo del banco de semillas de la especie Muhlenbergia peruviana, una gramínea anual de muy pequeño porte. Esta especie tendría un papel importante en la colonización de discontinuidades o "claros" de la cobertura vegetal producidos por las actividades de los herbívoros. La importancia de este tipo de especies pioneras a través de su "efecto fundador" en relación a la regeneración de la comunidad establecida ha sido puesta de manifiesto por Grime (1998).

\section{Banco de semillas y vegetación establecida}

La similitud entre el banco de semillas y la vegetación establecida fue muy baja en ambos tratamientos, coincidiendo con lo observado en otros pastizales templados dominados por especies perennes, donde vegetación y banco de semillas no guardan mucha relación (Thompson \& Grime 1979, Milberg 1995, Bakker et al. 1996, Funes et al. 2001). Por el contrario, en pastizales templados sujetos a perturbaciones (Warr et al. 1993), o dominados por especies anuales (Peco et al. 1998), se ha observado que la composición de la vegetación y el banco de semillas suelen parecerse mucho. La baja similitud observada en la composición florística de la vegetación establecida y el banco de semillas frente al pastoreo, sugieren nuevamente que éste no se estaría comportando como una perturbación severa en los pastizales estudiados.

\section{Clasificación de los bancos de semillas}

En los pastizales estudiados, tanto en el tratamiento pastoreado como en el tratamiento excluido al pastoreo, predominaron las especies con banco de semillas transitorio, seguidas por especies con banco de semillas persistente a corto y a largo plazo. Estos resultados son coincidentes con los documentados por Bakker et al. (1996) y Funes et al. (2001) para pastizales templados. Por otra parte, el pastoreo produjo un aumento en la proporción de especies con banco de semillas persistente a largo plazo. Esto último, puede ser explicado por los movimientos de suelo provocados por el pisoteo del ganado, lo que favorece el enterramiento de semillas (McDonald et al. 1996). Estas perturbaciones sobre el suelo también explicarían el cambio de estrategia de algunas especies entre sitios excluidos al ganado y sitios pastoreados. Cambios en la estrategia de banco de semillas se han observado en algunas especies bajo diferentes condiciones microambientales en diferentes sistemas de montaña (Ortega et al. 1997, Cavieres \& Arroyo 1999, Funes et al. 2001).

En el sistema de pastizales naturales estudiado, el pastoreo por ganado doméstico, no promueve cambios sustanciales en la identidad de las especies presentes en la vegetación establecida, pero promueve la diversidad de especies. El pastoreo, por otro lado, no provoca cambios importantes en la composición y en la densidad del banco de semillas de la mayoría de las especies, pero produce un incremento en la abundancia de semillas de especies con bancos persistentes, importantes para la regeneración del pastizal luego de perturbaciones severas.

\section{AGRADECIMIENTOS}

Este trabajo pudo realizarse gracias a la desinteresada colaboración de Sandra Basconcelo y Mariana Nai Bregaglio en tareas de campo y laboratorio. Agradecemos al Consejo de Investigaciones Científicas y Tecnológicas de la Provincia de Córdoba (CONICOR) por los fondos suministrados para la realización del proyecto (Subs. No. 4366/97 a E. Pucheta), al Instituto Multidisciplinario de Biología Vegetal (IMBIV) y a la Universidad Nacional de Córdoba, por su apoyo institucional. 


\section{LITERATURA CITADA}

BAKKER JP, ES BAKKER, E ROSEN, GL VERGUEIJ \& RM BEKKER (1996) Soil seed bank composition along a gradient from dry alvar grassland to Juniperus shrubland. Journal of Vegetation Science 7: 165-176.

BELSKY AJ (1992) Effects of grazing, competition, disturbance and fire on species composition and diversity in grassland communities. Journal of Vegetation Science 3: 187-200.

BERTILLER MB (1992) Seasonal variation in the seed bank of a Patagonian grassland in relation to grazing and topography. Journal of Vegetation Science 3: 4754.

BERTILLER MB (1996) Grazing effects on sustainable semiarid rangelands in Patagonia: the stage and dynamics of the soil seed bank. Environmental Management 20: 123-132.

BERTILLER MB \& D ALOIA (1997) Seed bank strategies in Patagonian semi-arid grasslands in relation to their management and conservation. Biodiversity and Conservation 6: 639-650.

CABIDO M, R BREIMER \& G VEGA (1987) Plant communities and associated soil types in a high plateau of the Córdoba mountains, central Argentina. Mountain Research and Development 7: 25-42.

CABIDO M, G FUNES, E PUCHETA, F VENDRAMINI \& S DÍAZ (1998) A chorological analysis of the mountains from central Argentina: is all what we call Sierra Chaco really Chaco?. Contribution to the study of the flora and vegetation of the Chaco XII. Candollea 53: $321-331$.

CAVIERES LA \& MTK ARROYO (1999) Bancos de semillas en Phacelia secunda J.F. Gmelin (Hydrophyllaceae): variación altitudinal en los Andes de Chile central ( $\left.33^{\circ} \mathrm{S}\right)$. Revista Chilena de Historia Natural 72: 569-577.

CHANETON EJ \& JM FACELLI (1991) Disturbance effects on plant community diversity: spatial scales and dominance hierarchies. Vegetatio 93: 143-155.

DÍAZ S, A ACOSTA \& M CABIDO (1994a) Community structure in montane grasslands of central Argentina in relation to land use. Journal of Vegetation Science 5: 483-488.

DÍAZ S, A ACOSTA \& M CABIDO (1994b) Grazing and the phenology of flowering and fruiting in the montane grassland in Argentina: a niche approach. Oikos 70: 287-295.

DONELAN M \& K THOMPSON (1980) Distribution of buried viable seeds along successional series. Biological Conservation 17: 297-311.

FUNES G \& M CABIDO (1995) Variabilidad local y regional de la vegetación rupícola de las sierras grandes de Córdoba, Argentina. Kurtziana 24: 173-188.

FUNES G, S BASCONCELO, S DÍAZ \& M CABIDO (1999) Seed bank dynamics of Lachemilla pinnata (Rosaceae) in different plant communities of mountain grasslands in central Argentina. Annales Botanici Fennici 36: 109-114.

FUNES G, S BASCONCELO, S DÍAZ \& M CABIDO (2001) Edaphic patchiness influences grassland regeneration from the soil seed bank in mountain grasslands of central Argentina. Austral Ecology 26: 205-212.
GIBSON CWD \& VK BROWN (1991) The effects of grazing on local colonization and extinction during early succession. Journal of Vegetation Science 2: 291-300.

GILFEDDER L \& JB KIRKPATRICK (1993) Germinable seed bank and competitive relationships between a rare native species and exotics in a semi-natural pasture in the midlands, Tasmania. Biological Conservation 64: 113-119.

GRAHAN DJ \& MJ HUTCHINGS (1988) Estimation of the seed bank of the chalk grassland ley established on former arable land. Journal of Applied Ecology 25: 241-252.

GRIME JP (1979) Plant strategies and vegetation processes. John Wiley \& Sons, New York, New York. 222 pp.

GRIME JP (1998) Benefits of plant diversity to ecosystems: immediate, filter and founder effects. Journal of Ecology 86: 902-910.

HILL MO \& HG GAUCH (1980) Detrented correspondence analysis: an improved ordination technique. Vegetatio 42: 47-58.

JUTILA H (1998) Seed banks of grazed and ungrazed Baltic seashore meadows. Journal of Vegetation Science 9: 395-408.

KINUKAN RJ \& FE SMEINS (1992) Soil seed bank of the semiarid Texas grassland under 3 long-term (36-years) grazing regimes. American Midland Naturalist 128: 11-21.

KREBS CJ (1985) Ecología: estudio de la distribución y la abundancia. Harper \& Row Latinoamericana, Ciudad de México, México. 654 pp.

LEPS J, J MICHÁLEK, P KULISEK \& P UHLÍK (1995) Use of paired plots and multivariate analysis for the determination of goat grazing preference. Journal of Vegetation Science 6: 37-42.

LUTI R, M SOLÍS, FM GALERA, N MÜLLER, M BERZAL, M NORES, M HERRERA \& JC BARRERA (1979) Vegetación. En: Vázquez J, R Miatello \& M Roqué (eds) Geografía física de la Provincia de Córdoba: 297-368. Ediciones Boldt, Buenos Aires, Argentina.

MCDONALD A, JP BAKKER \& K VEGELIN (1996) Seed bank classification and its importance for the restoration of species-rich flood-meadows. Journal of Vegetation Science 7: 157-164.

MCNAUGHTON SJ (1979) Grazing as an optimization process: grass-ungulate relationships in the Serengeti. American Naturalist 113: 691 -703.

MILBERG P (1995) Soil seed bank after eighteen years succession from grassland to forest. Oikos 72: 3-13.

MILBERG P \& ML HANSSON (1993) Soil seed bank and species turnover in a limestone grassland. Journal of Vegetation Science 4: 35-42.

MILCHUNAS DG \& WK LAUENROTH (1993) A quantitative assessment of the effects of grazing on vegetation and soils over a global range of environments. Ecological Monographs 63: 327-366.

MILCHUNAS DG, OE SALA \& WK LAUENROTH (1988) A generalized model of the effects of grazing by large herbivores on grassland community structure. American Naturalist 132: 87-106.

NORUSIS MJ (1992) SPSS for Windows. Advanced Statistics Release 5. SPSS Inc., Chicago, Illinois. 580 pp. 
NOY-MEIR I, M GUTMAN \& Y KAPLAN (1989) Responses of Mediterranean grassland plants to grazing and protection. Journal of Ecology 77: 290310.

O'CONNOR TG (1991) Local extinction in perennial grasslands: a life-history approach. American Naturalist 137: 753-773.

O'CONNOR TG \& STA PICKETT (1992) The influence of grazing on seed production and seed banks of some African savanna grasslands. Journal of Applied Ecology 29: 247-260.

OESTERHELD M \& OE SALA (1990) Effects of grazing on seedling establishment: the role of seed and safesite availability. Journal of Vegetation Science 1: 353- 358.

ORTEGA M, C LEVASSOR \& B PECO (1997) Seasonal dynamics of Mediterranean pasture seed banks along environmental gradients. Journal of Biogeography 24: 177-95.

PANDEY CB \& JS SINGH (1992) Influence of rainfall and grazing on herbage dynamics in a seasonally dry tropical savanna. Vegetatio 102: 107-124.

PECO B, M ORTEGA \& C LEVASSOR (1998) Similarity between seed bank and vegetation in Mediterranean grassland: a predictive model. Journal of Vegetation Science 9: 815-828.

PUCHETA E \& M CABIDO (1992) Comunidades de pastizales serranos del centro de Argentina y su relación con el uso pastoril. Phytocoenología 21: 333 346.

PUCHETA E, S DÍAZ \& M CABIDO (1992) The effect of grazing on the structure of a high plateau grassland in central Argentina. Coenoses 7: 145-152.

PUCHETA E, M CABIDO \& S DÍAZ (1997) Modelos de estados y transiciones para los pastizales de altura de las sierras de Córdoba, Argentina. Ecotrópicos 10: 151-160.

PUCHETA E, M CABIDO, S DÍAZ \& G FUNES (1998a) Floristic composition, biomass and aboveground net plant production in grazed and protected sites in mountain grassland of central Argentina. Acta Ecologica 19: 97-105.

PUCHETA E, F VENDRAMINI, M CABIDO \& S DÍAZ (1998b) Estructura y funcionamiento de un pastizal de montaña bajo pastoreo y su respuesta luego de su exclusión. Revista de la Facultad de Agronomía de La Plata (Argentina) 103: 77-92.

REDMANN RE (1992) Primary productivity. En: Coupland RT (ed) Natural grasslands: introduction and Western Hemisphere: 75-93. Elsevier, New York, New York. SALA OE, M OESTERHELD, RJC LEÓN \& A SORIANO (1986) Grazing effects upon plant community structure in subhumid grassland of Argentina. Vegetatio 67: 27-32.
SALA OE, VA DEREGIBUS, TM SCHLICHTER \& H ALIPPE (1981) Productivity dynamics of a native temperate grassland in Argentina. Journal of Range Management 34: 48-51.

SIPOWICZ DI, R LUTI \& C MORLANS (1978) Productividad primaria de la estepa de altura de las Sierras Grandes, Córdoba. Ecología Argentina 3: 117-123.

SOKAL RR \& FJ ROHLF (1995) Biometry. Freeman, San Fransisco, California. 887 pp.

TER HEERDT GN, GL VERWEIS, RM BAKKER \& JP BAKKER (1996) An improved method for seed banks analysis: emergence after removing the soil by sieving. Functional Ecology 10: 144-151.

THOMPSON K (1986) Small-scale heterogeneity in the seed bank of an acidic grassland. Journal of Ecology 74: 733-738.

THOMPSON K \& JP GRIME (1979) Seasonal variation in the soil seed banks of herbaceous species in ten contrasting habitats. Journal of Ecology 67: 893-921.

THOMPSON K, J BAKKER \& R BEKKER (1997) The soil seed banks of north west Europe: methodology, density and longevity. Cambridge University Press, Cambridge, United Kingdom. 276 pp.

UNGAR IA \& SRJ WOODELL (1996) Similarity of seed banks to aboveground vegetation in grazed and ungrazed salt marsh communities on the Gover Peninsula, South Wales. International Journal of Plant Science 157: 746-749.

VAN DER VALK AG \& L PEDERSON (1989) Seed banks and management and restoration of natural vegetation. En: Leck MA, VT Parker \& RL Simpson (eds) Ecology of seed banks: 329-346. Academic Press, London, Inglaterra.

WARR SJ, K THOMPSON \& M KENT (1993) Seed banks as a neglected area of biogeografic research: a review of literature and sampling techniques. Progress in Physical Geography 17: 329-347.

WATT TA \& CWD GIBSON (1988) The effects of the sheep grazing on seedling establishment and survival in grassland. Vegetatio 78: 91-98.

WILSON AD (1990) The effect of grazing on Australian ecosystems. Proceedings of the Ecological Society of Australia 16: 235-244.

ZULOAGA FO \& O MORRONE (1996) Catálogo de las plantas vasculares de la República Argentina. I. Monographs in Systematic Botany from the Missouri Botanical Garden 60: 1-323.

ZULOAGA FO \& O MORRONE (1999) Catálogo de las plantas vasculares de la República Argentina. II. Monographs in Systematic Botany from the Missouri Botanical Garden 74: 1-1269.

Editor Asociado: J. Gutiérrez

Recibido el 14 de diciembre de 2001; aceptado el 28 de enero de 2002 


\section{ANEXO 1}

Clasificación de las especies encontradas tanto en la vegetación establecida (V) como el banco de semillas (B) en este estudio en los sitios pastoreados (p) y excluidos (e) según el tipo de estrategia de banco de semillas que presenta. T: transitorio, PCP: persistente a corto plazo y

PLP: persistente a largo plazo

Classification of species found both in the standing vegetation (V) and in the seed bank (B) in grazed (p) and excluded (e) sites take into account the seed bank strategy. T: transient, PCP: short-term persistent and PLP: long-term persistent

Especie
Acicarpha tribuloides

Adesmia bicolor

Adesmia corymbosa

Agrostis montevidensis

Agrostis glabra v.glabra

Agrostis sp.

Alchemilla sp.

Cyclospermum leptophyllum

Aristida adscensionis

Baccharis tucumanensis

Bidens triplinervia

Briza subaristata

Bromus auleticus

Bromus brevis

Bulbostylis juncoides

Cardionema ramosissima

Carex boliviensis

Carex fuscula

Carex sp.

Castilleja pumila

Cerastium arvense

Cerastium glomeratum

Chaptalia integerrima

Chenopodium chilense

Cortaderia selloana

Cotula mexicana

Cyperus reflexus

Pycreus rivularis

Cyperus sp.

Daucus pusillus

Deyeuxia hieronymi

Dichondra sp.

Dicotiledónea no identificada.

Eleocharis albibracteata

Eragrostis lugens

Eryingium agavifolium

Eryngium nudicaule

Festuca tucumanica

Gamochaeta filaginea

Gamochaeta simplicicaulis

Gamochaeta coarctata

Gamochaeta subfalcata

Gentianella parviflora

Geranium magellanicum
Ocurrencia

$\mathrm{Ve} / \mathrm{Be}$
$\mathrm{Ve}-\mathrm{Vp}$
$\mathrm{Ve}-\mathrm{Vp}$
$\mathrm{Ve}-\mathrm{Vp}$
$\mathrm{Vp}$
$\mathrm{Vp}$
$\mathrm{Be}-\mathrm{Bp}$
$\mathrm{Be}-\mathrm{Bp}$
$\mathrm{Bp}$
$\mathrm{Ve}$
$\mathrm{Ve}-\mathrm{Vp}$
$\mathrm{Ve}-\mathrm{Vp}$
$\mathrm{Ve}$
$\mathrm{Vp}$
$\mathrm{Bp}$
$\mathrm{Vp}$
$\mathrm{Vp}$
$\mathrm{Ve}-\mathrm{Vp}$

Estrategia

Excluido Pastoreado

$\mathrm{Ve}-\mathrm{Vp} / \mathrm{Be}-\mathrm{Bp}$

$\mathrm{Vp}$

$\mathrm{Vp} / \mathrm{Be}$

$\mathrm{Be}$

Ve-Vp

$\mathrm{Be}$

$\mathrm{Be}$

$\mathrm{Be}$

Ve-Vp

$\mathrm{Vp} / \mathrm{Be}-\mathrm{Bp}$

$\mathrm{Bp}$

Be-Bp

$\mathrm{Ve}-\mathrm{Vp} / \mathrm{Be}-\mathrm{Bp}$

$\mathrm{Ve}-\mathrm{Vp}$

$\mathrm{Bp}$

$\mathrm{Vp} / \mathrm{Be}-\mathrm{Bp}$

$\mathrm{Ve}-\mathrm{Vp} / \mathrm{Be}-\mathrm{Bp}$

$\mathrm{Ve}-\mathrm{Vp} / \mathrm{Be}$

Ve-Vp/Be-Bp

$\mathrm{Ve}$

$\mathrm{Ve}-\mathrm{Vp} / \mathrm{Be}-\mathrm{Bp}$

Be-Bp

$\mathrm{Ve}-\mathrm{Vp} / \mathrm{Be}-\mathrm{Bp}$

$\mathrm{Be}$

$\mathrm{Ve}-\mathrm{Vp}$

Ve-Vp

$\begin{array}{cr}\mathrm{T} & \\ \mathrm{T} & \mathrm{T} \\ \mathrm{T} & \\ - & \mathrm{T} \\ - & \mathrm{T} \\ \mathrm{T} & \mathrm{P} \\ \mathrm{PCP} & \mathrm{PCP} \\ - & \mathrm{P} \\ \mathrm{T} & \\ \mathrm{T} & \mathrm{T} \\ \mathrm{T} & \mathrm{T} \\ \mathrm{T} & \\ - & \mathrm{T} \\ - & \mathrm{T} \\ - & \mathrm{T} \\ - & \mathrm{T} \\ \mathrm{T} & \mathrm{T}\end{array}$

PCP PCP

$\begin{array}{ll}- & T\end{array}$

PLP T

T -

T T

T -

T -

T -

T T

PCP PCP

- PLP

T T

PCP T

$\mathrm{T} T$

- T

PCP PLP

PLP PLP

T T

T T

T L

T PCP

PCP T

PCP PCP

T -

T T

T $\mathrm{T}$ 


\begin{tabular}{|c|c|c|c|}
\hline \multirow[t]{2}{*}{ Especie } & \multirow[t]{2}{*}{ Ocurrencia } & \multicolumn{2}{|c|}{ Estrategia } \\
\hline & & Excluido & Pastoreado \\
\hline Geranium patagonium & $\mathrm{Be}$ & $\mathrm{T}$ & - \\
\hline Gnaphalium gaudichaudianum & $\mathrm{Be}$ & PCP & - \\
\hline Grindelia globularifolia & $\mathrm{Ve}-\mathrm{Vp} / \mathrm{Be}$ & $\mathrm{T}$ & $\mathrm{T}$ \\
\hline Hieracium giganteum & Ve-Vp & $\mathrm{T}$ & $\mathrm{T}$ \\
\hline Hypoxis decumbens & $\mathrm{Be}$ & PLP & - \\
\hline Hypochaeris aff.argentina & $\mathrm{Ve}$ & $\mathrm{T}$ & - \\
\hline Juncus pallescens $v$. achalensis & $\mathrm{Ve}-\mathrm{Vp} / \mathrm{Be}-\mathrm{Bp}$ & PCP & PCP \\
\hline Juncus bufonius & Be-Bp & $\mathrm{T}$ & PCP \\
\hline Juncus dichotomus & $\mathrm{Be}-\mathrm{Bp}$ & PCP & $\mathrm{PCP}$ \\
\hline Juncus uruguensis & $\mathrm{Ve}-\mathrm{Vp}$ & $\mathrm{T}$ & $\mathrm{T}$ \\
\hline Alchemilla pinnata & $\mathrm{Ve}-\mathrm{Vp} / \mathrm{Be}-\mathrm{Bp}$ & PCP & PCP \\
\hline Linaria texana & Be-Bp & PLP & PLP \\
\hline Luzula excelsa & $\mathrm{Vp}$ & - & $\mathrm{T}$ \\
\hline Margyricarpus pinnatus & $\mathrm{Ve}-\mathrm{Vp}$ & $\mathrm{T}$ & $\mathrm{T}$ \\
\hline Mitracarpus megapotamicus & $\mathrm{Vp}$ & - & $\mathrm{T}$ \\
\hline Muhlenbergia ligularis & $\mathrm{Ve} / \mathrm{Bp}$ & $\mathrm{T}$ & PLP \\
\hline Muhlenbergia peruviana & $\mathrm{Vp} / \mathrm{Be}-\mathrm{Bp}$ & $\mathrm{T}$ & PCP \\
\hline Nothicastrum marginatum & $\mathrm{Ve}-\mathrm{Vp} / \mathrm{Be}$ & $\mathrm{T}$ & $\mathrm{T}$ \\
\hline Nothoscordum gracile & Ve-Vp & $\mathrm{T}$ & $\mathrm{T}$ \\
\hline Oenotera indecora & Ve-Vp & $\mathrm{T}$ & $\mathrm{T}$ \\
\hline Oreomyrrhis andicola & Ve-Vp & $\mathrm{T}$ & $\mathrm{T}$ \\
\hline Paspalum himboldtianum & $\mathrm{Bp}$ & - & PLP \\
\hline Pictochaetium montevidense & $\mathrm{Ve}-\mathrm{Vp}$ & $\mathrm{T}$ & $\mathrm{T}$ \\
\hline Plantago brasiliensis & Ve-Vp & $\mathrm{T}$ & $\mathrm{T}$ \\
\hline Plantago myosurus & $\mathrm{Vp}$ & - & $\mathrm{T}$ \\
\hline Plantago tomentosa & $\mathrm{Be}-\mathrm{Bp}$ & $\mathrm{T}$ & $\mathrm{T}$ \\
\hline Роа аппиа & $\mathrm{Vp}$ & - & $\mathrm{T}$ \\
\hline Poa resinulosa & $\mathrm{Ve}-\mathrm{Vp} / \mathrm{Bp}$ & $\mathrm{T}$ & $\mathrm{T}$ \\
\hline Poa stuckertii & $\mathrm{Ve} / \mathrm{Be}$ & $\mathrm{T}$ & - \\
\hline Pratia hederacea & $\mathrm{Vp}$ & - & $\mathrm{T}$ \\
\hline Ranunculus praemorsus & $\mathrm{Vp}$ & - & $\mathrm{T}$ \\
\hline Galium richardianum & $\mathrm{Ve}-\mathrm{Vp} / \mathrm{Bp}$ & $\mathrm{T}$ & $\mathrm{T}$ \\
\hline Rumex acetosella & $\mathrm{Ve}-\mathrm{Vp} / \mathrm{Be}$ & $\mathrm{T}$ & $\mathrm{T}$ \\
\hline Scoparia montevidensis & Bp & - & $\mathrm{T}$ \\
\hline Silene antirrhina & Bp & - & PLP \\
\hline Sorghastrum pellitum & $\mathrm{Ve}-\mathrm{Vp} / \mathrm{Be}$ & $\mathrm{T}$ & $\mathrm{T}$ \\
\hline Spergula ramosa & $\mathrm{Be}$ & PLP & - \\
\hline Sporobolus indicus & $\mathrm{Ve}-\mathrm{Vp} / \mathrm{Be}-\mathrm{Bp}$ & $\mathrm{T}$ & $\mathrm{T}$ \\
\hline Stenandrium dulce & Ve-Vp & $\mathrm{T}$ & $\mathrm{T}$ \\
\hline Stipa eriostachya & $\mathrm{Be}-\mathrm{Bp}$ & $\mathrm{T}$ & PLP \\
\hline Stipa juncoides & $\mathrm{Be}$ & $\mathrm{T}$ & - \\
\hline Stipa neesiana & $\mathrm{Be}$ & - & $\mathrm{T}$ \\
\hline Stipa nidulans & $\mathrm{Vp}$ & - & $\mathrm{T}$ \\
\hline Stipa tenuissima & $\mathrm{Ve}$ & $\mathrm{T}$ & - \\
\hline Sysirrinchium sp. & Bp & - & PCP \\
\hline Sisyrrinchum chilense & $\mathrm{Vp} / \mathrm{Be}-\mathrm{Bp}$ & PCP & PCP \\
\hline Tagetes filifolia & $\mathrm{Vp} / \mathrm{Bp}$ & - & $\mathrm{T}$ \\
\hline Tagetes minuta & $\mathrm{Be}$ & $\mathrm{T}$ & - \\
\hline Taraxacum officinale & $\mathrm{Ve}-\mathrm{Vp} / \mathrm{Be}-\mathrm{Bp}$ & $\mathrm{T}$ & PLP \\
\hline Trifolium sp. & Ve-Vp & $\mathrm{T}$ & $\mathrm{T}$ \\
\hline Triodanis perfoliata $v$. biflora & $\mathrm{Be}$ & $\mathrm{T}$ & - \\
\hline Veronica peregrina & $\mathrm{Be}-\mathrm{Bp}$ & PLP & PCP \\
\hline Vicia graminea & $\mathrm{Be}$ & $\mathrm{T}$ & - \\
\hline
\end{tabular}

